\title{
Assessment of the Relation between Fetal Hemodynamic Indices in Late Pregnancy and Birth Weight in Gestational Diabetic Mellitus Mothers: A correlational study
}

\author{
Magdy Mohamed Mahmoud Abd el Gawad ${ }^{1}$, Mohamed Hussain Moustafa ${ }^{\mathbf{1}}$, Laila Aly Farid ${ }^{\mathbf{1}}$, \\ Noha Emad El Din Abd El Aziz ${ }^{2}$
}

1. Obstetrics and Gynaecology Faculty of Medicine - Ain Shams University.

2. Obstetrics and Gynecology at Manshyet El Bakry General Hospital.

\begin{abstract}
Background: The offspring of women with gestational diabetes mellitus (GDM) are prone to macrosomia. However, birth weight is difficult to be correctly estimated by ultrasound because of fetal asymmetric growth characteristics. This study aimed at investigating the correlations between fetal hemodynamics, fetal growth indices in late pregnancy and birth weight in GDM. Methods: A total of 180 women with GDM and 180 normal controls (NC) with singleton gestation and presented between 38-40 weeks gestation were enrolled in this study. Fetal hemodynamic indices, including the systolic/diastolic ratio (S/D), resistance index (RI), pulsatility index (PI) of umbilical artery (UA), middle cerebral artery (MCA), and renal artery (RA), were collected. Fetal growth indices, including biparietal diameter (BPD), head circumference (HC), abdominal circumference (AC), and femur length (FL), were also measured by ultrasound. Birth weight, mode of delivery and need for Neonatal ICU admission data were collected. Results: The independent samples $t$-test showed that BPD, HC, $\mathrm{AC}$ and FL were larger in GDM than in NC $(P<0.05)$. Birth weight was higher in GDM than in NC $(P<0.001)$. Among all included women, there was a highly statistically insignificant difference between GDM and NC groups as regard all ultrasound indices including UA_S/D, UA_RI, UA_PI, MCA_S/D, MCA_RI, MCA_PI, RA_S/D, RA_RI and RA_PI (P>0.05). Pearson's correlation analysis showed in GDM group that there was a highly statistically significant negative correlation between birth weight and the following ultrasound indices: (UA_RI, UA_S/D, UA_PI, MCA_RI and MCA_PI) and that there was a statistically significant positive correlation between birth weight \& RA_RI $(\mathrm{P}<0.01)(r=-0.273,-0.453,-0.537,-0.237,-0.265$ and 0.169 respectively, $P<0.05)$, but As regard NC group there was a highly statistically significant negative correlation between birth weight and the following ultrasound indices: (UA_S/D, UA_RI, UA_PI and MCA_PI) $(r=0.148$, $0.360,-0.252$ and -0.184 respectively, $P<0.05$ ) but no correlation was found with any of renal artery indices $(P>0.05)$. Conclusions: Fetal hemodynamic indices in late pregnancy might be helpful for estimating newborn birth weight in women with GDM.
\end{abstract}

Keywords: Fetus; Gestational Diabetes Mellitus; Infant; Middle Cerebral Artery; Renal Artery; Ultrasound; Umbilical Artery.

\section{INTRODUCTION}

GDM is diabetes diagnosed during pregnancy that is not clearly type 1 or 2 diabetes ${ }^{(1)}$. Approximately $84 \%$ of hyperglycemia cases during pregnancy are due to GDM. The estimated incidence of GDM in Europe is $3 \%$ to $5 \%$ or 150,000 to 250,000 pregnant women out of the five million who give birth each year ${ }^{(2)}$. In Egypt, Impaired Glucose Tolerance which occurs during pregnancy affects $7.2 \%$ of all pregnancies and is considered a major cause of maternal and fetal morbidity ${ }^{(3)}$.
The most common and significant neonatal complication associated with GDM is macrosomia, which is defined as a birth weight greater than 4,000 to 4.500 grams, as well as larger for gestational age, with the birth weight above to $90^{\text {th }}$ percentile for population-specific and set-specific growth curves ${ }^{(4)}$. The increase in fetal body fat occurs in the insulin dependent tissues, such as the thighs, intrahepatic and abdominal adipose tissues. Fat accumulation tends to be truncal with larger shoulder circumference which leads to an increased risk for cephalopelvic disproportion shoulder dystocia, and birth trauma ${ }^{(5)}$. 
Sonographic-estimated fetal weight (EFW) is usually obtained from four standard sonographic measurements of the fetus: BPD, $\mathrm{HC}, \mathrm{AC}$, and FL. The Hadlock formula is one of the most widely used and precise formulas, and several studies have shown that it presents the smallest systematic mean error ${ }^{(6)}$. The accuracy of fetal weight estimation carries a margin of error of 5-10\% for those fetuses that are normal or small in size. The percentage of inaccuracy increases significantly in very large fetuses such that any EFW calculated to be greater than $3.8 \mathrm{~kg}$ should be interpreted with great caution ${ }^{(7)}$.

The umbilical artery (UA) is the major vascular pathway connecting the fetus and placenta. The fetus obtains nutrients and oxygen through the umbilical circulation. The systolic/ diastolic ratio (S/D), pulsatility index (PI), and resistance index (RI) are the hemodynamic indices of the fetoplacental circulation. The fetal middle cerebral artery (MCA) can directly reflect blood circulation of the fetal brain, and the S/D, PI, and RI are the hemodynamic indices of brain circulation. The fetal renal artery (RA) also tends to directly reflect blood perfusion of the fetal kidney. The $\mathrm{RA}$ is one of the organs sensitive to hypoxia and one of the first organs to have endothelial dysfunction ${ }^{(8-10)}$.

This study investigated the correlations among fetal hemodynamic indices (S/D, PI, and RI) of the UA, MCA, and RA, fetal growth in late pregnancy, and newborn birth weight in women with GDM and normal controls (NCs, normal pregnant women), with a view to determining whether fetal hemodynamic indices in late pregnancy can assist doctors in estimating newborn birth weight in GDM.

\section{METHODOLOGY}

This observational study was conducted at Ain Shams University Maternity Hospital in the period from September 2017 till April 2018. The Hospital's Research Ethics Committee approved the study protocol. The study included 360 pregnant women with presented between 38-40 weeks gestation divided into two groups, Group I : GDM group Including 180 pregnant controlled diabetic women and Group II : NC Group.
Inclusion criteria were singleton viable pregnancy, gestational age from 38 to 40 weeks, subjects age between 25-38 years wih body mass index between 30 and 34.9 $\mathrm{kg} / \mathrm{m} 2$. GDM group had controlled DM checked by measuring fasting plasma glucose (FPG) concentration which was less than $140 \mathrm{mg} / \mathrm{dl}$ and $\mathrm{HbA} 1 \mathrm{c}$ below $6.5 \%$. Exclusion criteria were multiple gestations, gestational age before 38 weeks or more than 40 weeks, subjects with body mass index less than 30 or more than $34.9 \mathrm{~kg} / \mathrm{m} 2$, past history of DM before pregnancy, other well known condition affecting fetal blood flow, such as intrauterine growth restriction, anemia, hypoxemia, pregnancy induced hypertension or renal disorders, previous history of a newborn with congenital anomalies or in current pregnancy, mothers with uncontrolled GDM, history of hyperlipidemia, and smoking. The diagnostic thresholds for GDM were according to the ADA in 2016 during a two-hour oral glucose tolerance test (OGTT): glucose levels of 95, 140 and $120 \mathrm{mg} / \mathrm{dl}$ for fasting, one-hour and two-hour respectively post $75 \mathrm{~g}$ glucose. The criteria for the diagnosis of gestational induced hypertension issued by the World Health Organization were systolic blood pressure $\geq 140 \mathrm{mmHg}$ and/or diastolic blood pressure $\geq 90 \mathrm{mmHg}{ }^{(11)}$. All subjects undergone examination including general, abdominal and vaginal examination, were investigated for fasting blood glucose level, two hour post prandial blood glucose level and $\mathrm{Hb} \mathrm{A} 1 \mathrm{C}$ was done for diabetic group.

\section{Ultrasound measurements :} Transabdominal 2D ultrasound was done for all subjects to detect fetal heart rate, fetal presentation, amniotic fluid index and placental site with exclusion of congenital fetal malformations. The BPD was measured from the outer edge of the parietal bone near the probe to the inner edge of the other side of the parietal bone in the thalamencephalon, $\mathrm{HC}$ was measured in the same location as the $\mathrm{BPD}, \mathrm{AC}$ was measured along the outer layer of the skin in the area including the spine, gastric vacuole, and umbilical vein, The FL was measured at the center of the two ends of 
the femur, EFW was calculated using Hadlock's formula ${ }^{(12,13)}$.

Doppler Ultrasound assessment: The color flow pattern was selected to measure hemodynamic parameters of the UA, MCA, and RA. Measurements were performed at the UA within $5 \mathrm{~cm}$ from the placenta, during which the angle between the ultrasound beam and blood flow was adjusted to $<20^{\circ}{ }^{(14)}$. For the MCA, in the standard plane for BPD measurement, the probe was moved toward the brain basement membrane until a pair of alisphenoids was visible between the anterior and middle cranial fossa. An additional Doppler spectrum was then be applied to reveal the circle of Willis. The sampling volume was placed slightly before the middle part of the MCA, and the angle of the ultrasound beam and blood flow was adjusted to $<20^{\circ}{ }^{(15)}$. [Figure 1] For the RA, measurements were performed at a location close to the renal hilum and the angle of the ultrasound beam then blood flow was adjusted to $<20^{\circ}{ }^{(16)}$. The apparatus used was DCN3 Mindray ultrasound machine with Doppler unit and comvex linear transducer 3.5 MHZ. One senior sonographer undergone the sonography to eliminate any interpersonal errors and all measurements were taken twice and the mean of each value was taken to decrease intrapersonal errors.

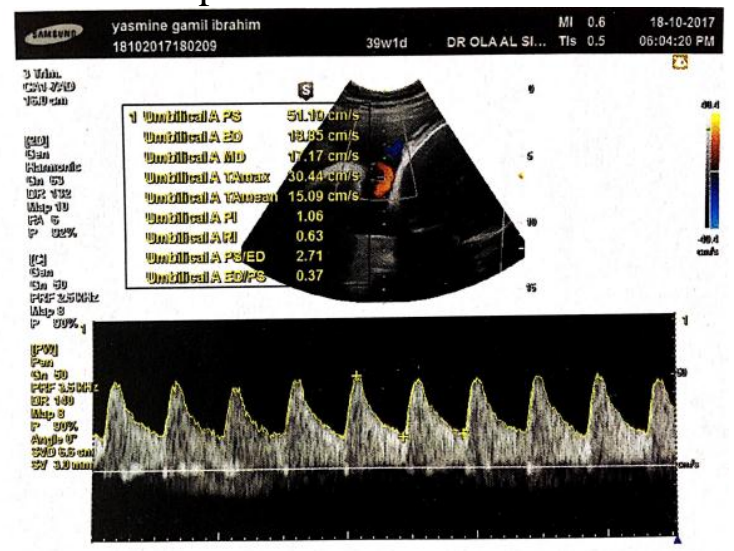

(a)

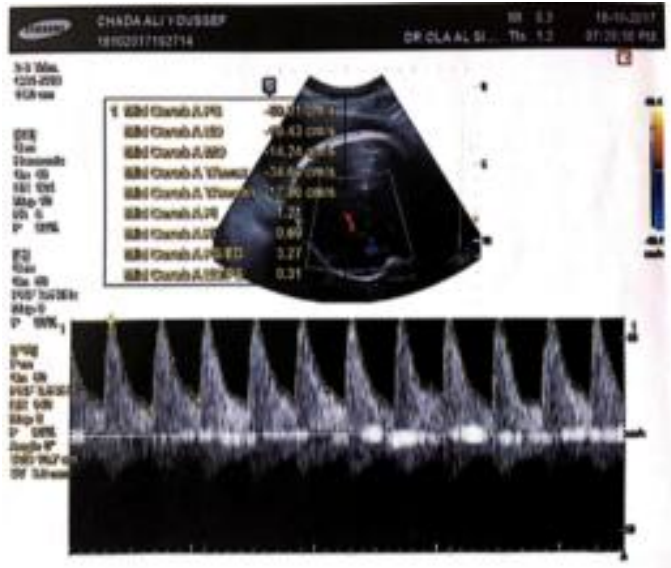

(b)

Figure 1: (a) Ultrasound Doppler spectrum of UA; (b) Ultrasound Doppler spectrum of MCA; UA: Umbilical Artery; MCA: Middle Cerebral Artery; S/D: Systolic/diastolic ratio; PI: Pulsatility index; RI: Resistance index.

Statistical analysis: Data were analyzed using SPSS version 17.0 software (SPSS Inc., Chicago, IL, USA). Measurement data were presented as mean \pm standard deviation and count data were expressed as $\mathrm{n}(\%)$. The independent samples t-test was used to compare the mean of continuous variables. The chi square test was used as appropriate for comparing characteristics between the two groups. Pearson's correlation coefficient was used to estimate the correlations among the hemodynamic indices (S/D, PI, and RI) of the fetal UA, MCA, and RA in late pregnancy, fetal growth indices (BPD, HC, $\mathrm{AC}$, and FL) and birth weight. A difference of $\mathrm{P}<0.05$ is considered statistically significant.

\section{RESULTS}

Maternal clinical data were not significantly different between the two groups s regard age, systolic BP, diastolic $\mathrm{BP}, \mathrm{BMI}$ and gestational age $(P>0.05)$ as shown in table (1). The independent samples $t$-test showed that BPD, HC, AC and FL were larger in GDM than in NC $(P$ $<0.05)$ where the mean BPD in GDM group was $9.29 \pm 0.23 \mathrm{~mm}$ and in $\mathrm{NC}$ group was $9.08 \pm 0.32 \mathrm{~mm}$. The mean $\mathrm{HC}$ 
in GDM group was $33.14 \pm 0.67 \mathrm{~mm}$ and in $\mathrm{NC}$ group was $32.56 \pm 0.84 \mathrm{~mm}$. The mean $\mathrm{AC}$ in GDM group was $34.51 \pm 1.24 \mathrm{~mm}$ and in NC group was $32.87 \pm 1.01 \mathrm{~mm}$. While the mean FL in GDM group was $7.56 \pm 0.21 \mathrm{~mm}$ and in NC group was $7.20 \pm 0.28 \mathrm{~mm}$. The mean EFW, using Hadlock's formula, was $3552.03 \pm 250.22 \mathrm{~g}$ in GDM group and 3065.29 $\pm 230.46 \mathrm{~g}$ in NC group showing also a highly statistically significant difference $(\mathrm{P}<0.01)$ as shown in table (2).
The mean birth weight in neonates

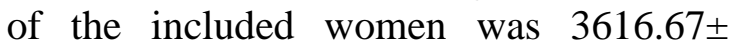
$202.08 \mathrm{~g}$ in GDM group and 3169.44 \pm $154.30 \mathrm{~g}$ in NC group showing a highly statistically significant difference as shown in table (1).

Among all included women, there was a highly statistically insignificant difference between both groups as regard all ultrasound indices including UA_S/D, UA_RI, UA_PI, MCA_S/D, MCA_RI, MCA_PI, RA_S/D, RA_RI and RA_PI $(\mathrm{P}>0.05)$ [Table 3].

Table 1: Comparison of descriptive data of the mothers, fetuses, and newborns in the GDM and NC groups (mean \pm SD)

\begin{tabular}{|c|c|c|c|}
\hline Variable & GDM $(n=180)$ & NC $(n=180)$ & $\mathbf{P}$ \\
\hline \multicolumn{4}{|l|}{ Mothers } \\
\hline Maternal age (years) & $30.84 \pm 2.79$ & $30.81 \pm 4.61$ & 0.934 \\
\hline Gestational age (weeks) & $38.14 \pm 0.35$ & $38.14 \pm 0.45$ & 1.000 \\
\hline BMI $\left(\mathrm{kg} / \mathrm{m}^{2}\right)$ & $33.33 \pm 3.36$ & $33.17+0.55$ & 0.541 \\
\hline $\begin{array}{l}\text { Systolic blood pressure } \\
(\mathrm{mmHg})\end{array}$ & $114.1 \overline{1+}+5.37$ & $113.22+4.69$ & 0.095 \\
\hline $\begin{array}{c}\text { Diastolic blood pressure } \\
(\mathrm{mmHg})\end{array}$ & $73.89+4.89$ & $73.22+4.69$ & 0.187 \\
\hline \multicolumn{4}{|l|}{ Fetuses } \\
\hline $\mathrm{BPD}(\mathrm{mm})$ & $9.29+0.23$ & $9.08+0.32$ & $<0.001 * *$ \\
\hline $\mathrm{HC}(\mathrm{mm})$ & $33.14+0.67$ & $32.56+0.84$ & $<0.001 * *$ \\
\hline $\mathrm{AC}(\mathrm{mm})$ & $34.51+1.24$ & $32.87+1.01$ & $<0.001 * *$ \\
\hline FL (mm) & $7.56+0.21$ & $7.20+0.28$ & $<0.001 * *$ \\
\hline UA S/D & $2.27+0.34$ & $2.28+0.34$ & $-0.391 \pm 0.696$ \\
\hline UA PI & $0.81+0.19$ & $0.81 \pm 0.19$ & $0.233 \pm 0.816$ \\
\hline UA RI & $0.56+0.05$ & $0.56+0.06$ & $-0.113+0.910$ \\
\hline MCA S/D & $3.94+1.10$ & $3.79 \pm 0.77$ & $1.470 \pm 0.142$ \\
\hline MCA PI & $1.44+0.29$ & $1.43+0.26$ & $0.378+0.705$ \\
\hline MCA RI & $0.78 \pm 0.10$ & $0.78+0.08$ & $0.067+0.947$ \\
\hline RA S/D & $6.08+1.76$ & $6.27 \pm 1.32$ & $-1.156+0.249$ \\
\hline RA PI & $2.00+0.41$ & $2.02+0.37$ & $-0.540+0.590$ \\
\hline RA RI & $0.94+0.91$ & $0.83 \pm 0.03$ & $1.706 \pm 0.089$ \\
\hline \multicolumn{4}{|l|}{ New borns } \\
\hline Birth weight & $3616.67 \pm 202.08$ & $3169.44 \pm 154.30$ & $<0.001 * *$ \\
\hline
\end{tabular}

(**) Highly statistically significant at $P<0.01$ 
Table (2): BPD, HC, AC, FL and EFW in women included in this study :

\begin{tabular}{|c|c|c|c|c|}
\hline \multirow{3}{*}{ variable } & \multicolumn{2}{|c|}{ Group } & \multirow{3}{*}{$\begin{array}{l}\text { Independent } \\
\text { Sample t-test }\end{array}$} & \multirow{3}{*}{$\begin{array}{l}\text { P-value and } \\
\text { significance }\end{array}$} \\
\hline & GDM & $\mathrm{NC}$ & & \\
\hline & Mean \pm SD & Mean \pm SD & & \\
\hline BPD & $9.29 \pm 0.23$ & $9.08 \pm 0.32$ & 7.168 & $<0.001 * *$ \\
\hline $\mathrm{HC}$ & $33.14 \pm 0.67$ & $32.56 \pm 0.84$ & 7.221 & $<0.001 * *$ \\
\hline $\mathrm{AC}$ & $34.51 \pm 1.24$ & $32.87 \pm 1.01$ & 13.681 & $<0.001 * *$ \\
\hline FL & $7.56 \pm 0.21$ & $7.20 \pm 0.28$ & 13.610 & $<0.001 * *$ \\
\hline EFW & $3552.03+250.22$ & $3065.29+230.46$ & 19.197 & $<0.001 * *$ \\
\hline
\end{tabular}

Table (3): UA, MCA and RA, S/D, PI and RI in women included in this study:

\begin{tabular}{|c|c|c|c|}
\hline \multicolumn{4}{|c|}{ Group } \\
\hline variable & $\begin{array}{c}\text { Gestational Diabetes } \\
\text { Mean } \pm \text { SD }\end{array}$ & $\begin{array}{c}\text { Non Diabetic } \\
\text { Mean } \pm \text { SD }\end{array}$ & $\begin{array}{l}\text { Independent } \\
\text { Sample t-test }\end{array}$ \\
\hline UA_S/D & $2.27 \pm 0.34$ & $2.28 \pm 0.34$ & $\begin{array}{c}-0.391 \pm 0.696 \\
\mathrm{NS}\end{array}$ \\
\hline UA_RI & $0.56 \pm 0.05$ & $0.56 \pm 0.06$ & $\begin{array}{c}-0.113 \pm 0.910 \\
\mathrm{NS}\end{array}$ \\
\hline UA_PI & $0.81 \pm 0.19$ & $0.81 \pm 0.19$ & $\begin{array}{c}0.233 \pm 0.816 \\
\mathrm{NS}\end{array}$ \\
\hline MCA_S/D & $3.94 \pm 1.10$ & $3.79 \pm 0.77$ & $\begin{array}{c}1.470 \pm 0.142 \\
\mathrm{NS}\end{array}$ \\
\hline MCA_RI & $0.78 \pm 0.10$ & $0.78 \pm 0.08$ & $\begin{array}{c}0.067 \pm 0.947 \\
\mathrm{NS}\end{array}$ \\
\hline MCA_PI & $1.44 \pm 0.29$ & $1.43 \pm 0.26$ & $\begin{array}{c}0.378 \pm 0.705 \\
\mathrm{NS}\end{array}$ \\
\hline RA_S/D & $6.08 \pm 1.76$ & $6.27 \pm 1.32$ & $\begin{array}{c}-1.156 \pm 0.249 \\
\text { NS }\end{array}$ \\
\hline RA_RI & $0.94 \pm 0.91$ & $0.83 \pm 0.03$ & $\begin{array}{c}1.706 \pm 0.089 \\
\mathrm{NS}\end{array}$ \\
\hline RA_PI & $2.00 \pm 0.41$ & $2.02 \pm 0.37$ & $\begin{array}{c}-0.540 \pm 0.590 \\
\mathrm{NS}\end{array}$ \\
\hline
\end{tabular}

$\overline{N S: \text { Non significant }}$

\section{Correlational Analysis:}

In both groups $\mathrm{BPD}, \mathrm{HC}, \mathrm{AC}$ and $\mathrm{FL}$ were positively correlated with birth weight, where in GDM group ( $\mathrm{r}=0.287,0.292$, 0.695 , and 0.472 , respectively, all $\mathrm{P}<0.05)$ and in NC group, $(\mathrm{r}=0.379,0.377,0.685$, and 0.594 , respectively, all $\mathrm{P}<0.05)$
[Table 4]. In the GDM group, there was a highly statistically significant negative correlation between birth weight and ultrasound indices (UA_RI, UA_S/D, UA_PI, MCA_RI \& MCA_PI) and there was a statistically significant positive correlation between birth weight and RA_RI in $(\mathrm{P}<0.01)$. However; in $\mathrm{NC}$ 
group there was a highly statistically significant negative correlation between birth weight and ultrasound indices (UA_S/D, UA_RI, UA_PI \& MCA_PI)
$(\mathrm{P}<0.01)$ while no statistically significant correlation was found between birth weight and RA_RI in NC group [Table 5].

Table (4): Shows correlation between Birth Weight and Fetal growth indices in both groups:

\begin{tabular}{|c|c|c|c|c|}
\hline \multirow{3}{*}{ Variables } & \multicolumn{4}{|c|}{ Birth Weight } \\
\hline & GDM & & $\mathrm{NC}$ & \\
\hline & $\mathbf{r}$ & $\mathbf{p}$ & $\mathbf{r}$ & $\mathbf{p}$ \\
\hline BPD & 0.287 & $<0.001 * *$ & 0.379 & $<0.001 * *$ \\
\hline Head Circumference & 0.292 & $<0.001 * *$ & 0.377 & $<0.001 * *$ \\
\hline $\mathrm{AC}$ & 0.695 & $<0.001 * *$ & 0.685 & $<0.001 * *$ \\
\hline FL & 0.472 & $<0.001 * *$ & 0.594 & $<0.001 * *$ \\
\hline
\end{tabular}

(**) Highly statistically significant at $P<0.01$

Table (5) : Shows correlation between Birth Weight and Ultrasound indices in both groups :

\begin{tabular}{lcccc}
\hline Variables & \multicolumn{2}{c}{ Birth Weight } & \multicolumn{2}{c}{ NC } \\
& GDM & r & p & p \\
\hline UA_S/D & -0.453 & $<0.001^{* *}$ & -0.148 & $0.047^{*}$ \\
UA_RI & -0.273 & $<0.001^{* *}$ & -0.360 & $<0.001^{* *}$ \\
UA_PI & -0.537 & $<0.001^{* *}$ & -0.252 & $0.001^{* *}$ \\
MCA_SD & -0.138 & 0.065 & -0.096 & 0.202 \\
MCA_RI & -0.237 & $0.001^{* *}$ & -0.009 & 0.906 \\
MCA_PI & -0.265 & $<0.001^{* *}$ & -0.184 & $0.014^{*}$ \\
RA_S/D & 0.056 & 0.453 & 0.079 & 0.294 \\
RA_RI & 0.169 & $0.023^{*}$ & 0.079 & 0.290 \\
RA_PI & 0.056 & 0.455 & 0.007 & 0.921 \\
\hline
\end{tabular}

(*) Satistically significant at $P<0.05$

(**) Highly statistically significant at $P<0.01$

\section{Discussion}

DM induced newborn and maternal complications include fetal death, fetal malformation, preeclampsia, intrauterine growth restriction, and fetal macrosomia. The incidence of fetal macrosomia ranges between $20 \%$ and $40 \%{ }^{(12)}$. Fat accumulation tends to be truncal with larger shoulder circumference which leads to an increased risk for cephalopelvic disproportion shoulder dystocia, and birth trauma ${ }^{(17)}$. The Hadlock formula is one of the most widely used and precise formulas for fetal weight estimation, and several studies have shown that it presents the smallest systematic mean error. However, birth weight is often inaccurately estimated using ultrasound in gestational diabetic mellitus mothers in late pregnancy because of fetal asymmetric growth characteristics ${ }^{(\mathbf{1 8 , 1 9 )}}$.

This prospective correlational study was designed to further investigate the correlations among fetal hemodynamic indices (S/D, PI, 
and RI of the UA, MCA, and RA) in late pregnancy, fetal growth indices, and newborn birth weight in GDM group and normal control group. Hyperglycemia in pregnant women with GDM tends to increase fetal growth through a series of pathophysiological responses only in late pregnancy ${ }^{(20)}$. To meet the requirements of fetal growth, placental blood perfusion and blood volume of the UA increase, and vascular resistance decreases, thus in GDM group, there was a highly statistically significant negative correlation between birth weight and ultrasound indices of UA (S/D, RI, and PI). The fetus tends to experience hypoxia and ischemia occurs when demand exceeds supply. A brain sparing effect is then triggered, leading to dilation of the MCA, which provides $80 \%$ of the blood supply to the cerebral hemisphere ${ }^{(21,15)}$. Resistance is reduced and brain development is promoted, so reflecting the statistically significant negative correlation found between birth weight and MCA_RI \& MCA_PI. Meanwhile, the RA, which is extremely sensitive to hypoxia and ischemia, tends to contract to redistribute blood flow. Thus a statistically significant positive correlation between birth weight and RA_RI in $(\mathrm{P}<0.01)$ was found in GDM group in order to ensure the blood supply to major organs of the fetus, such as the brain and liver.

A study made by Liu et al. in 2016 on 147 women with GDM and $124 \mathrm{NC}$ showed that BPD, HC, and AC were larger in GDM than in NC $(P<0.05)$. Fetal hemodynamic indices of the UA and MCA were lower $(P<$ $0.05)$, but those of the RA were higher $(P<$ $0.001)$ in GDM than in NC. Birth weight was higher in GDM than in NC $(P<0.001)$. Pearson's correlation analysis showed that hemodynamic indices of the UA were negatively correlated with birth weight in both groups $(P<0.05)$. MCA (S/D, PI, and RI) was negatively correlated with birth weight in GDM $(\mathrm{P}<0.05)$, but there were no correlations in NC $(P>0.05)$. RA (S/D, PI, and RI) was positively correlated with birth weight in GDM $(P<0.05)$, but there were no correlations in NC $(P>0.05)^{(\mathbf{1 2})}$.

A study made by Quintero-Prado et al. in 2014 on 169 women with gestational diabetes examining uterine artery PI and umbilical artery (UA) PI found a significant negative correlation between birthweight centiles and Z-score values of the UA-PI which is consistent with this study ${ }^{(22)}$.

A study made by Maruotti et al. in 2014 on 106 pregnant women with GDM found a descending relationship between UA PI and birth weight, where doppler recordings of UA-PI were performed at 34-41 weeks and related to neonatal birthweight. Linear regression analysis revealed a significant negative correlation between UA-PI and neonatal birthweight centile which is consistent with the current study ${ }^{(23)}$.

A study included 226 women with GDM showed that the umbilical artery hemodynamic indices (S/D, PI, and RI) in late pregnancy were strongly negatively correlated with birth weight which is consistent with the current study ${ }^{(16)}$.

A study made by Verburg et al. in 2008, also showed that UA PI was negatively correlated with estimated fetal weight which is consistent with the current study ${ }^{(24)}$.

\section{CONCLUSION}

In conclusion findings in the current study, in addition to data from previous studies, suggests that in diabetic group, there was a negative correlation between birth weight and UA_RI, UA_PI, UA_S/D, MCA_RI and MCA_PI. Also a positive correlation between RA_RI and birth weight was found. While in normal control group a negative correlation was found between birth weight and UA_S/D, UA_RI, UA_PI, and MCA-PI but no correlation was found with any of RA indices. Thus fetal hemodynamic indices in late pregnancy might be helpful for estimating newborn birth weight in women with GDM.

However, Further prospective trials on larger populations or groups with a higher prevalence of large for gestational age fetuses would be needed to validate a formula which can correlate UA, MCA and RA hemodynamic indices with birth weight in GDM mothers.

\section{Acknowledgements}

We are grateful to all of the patients who agreed to participate in the study. We are also grateful dr. Ola El Sirgany and to all staff members of Fetal Care Unit in Ain Shams University Maternity hospital for their contribution in this research. 


\section{References}

1- American Diabetes Association (2016): Standards of medical care in diabetes2016. Diabetes Care, 39(1):S1-S106.

2- Hod M, Kapur A, Sacks DA, Hadar E, Agarwal M, Di Renzo GC, Roura LC, McIntyre HD, Morris JL and Divakar H (2015): The International Federation of Gynecology and Obstetrics (FIGO) initiative on gestational diabetes mellitus: a pragmatic guide for diagnosis, management, and care. International Journal of Gynecology \& Obstetrics, 131(S3).

3- Azmy O, Mohsen M, Hassanain A, Abou-Ismail L and Habib D (2005): Influence of Oxidative Stress and Inflammatory Cytokines on Impaired Glucose Tolerance during Pregnancy and Neonatal Outcome. Med. J.N.R.C., 6 (1): 159.

4- Coustan DR, Lowe LP, Metzger BE and Dyer AR (2010): The Hyperglycemia and Adverse Pregnancy Outcome (HAPO) study: paving the way for new diagnostic criteria for gestational diabetes mellitus. American Journal of Obstetrics \& Gynecology, 202(6):654-e1.

5- Esakoff TF, Cheng YW, Sparks TN and Caughey AB (2009): The association between birthweight $4000 \mathrm{~g}$ or greater and perinatal outcomes in patients with and without gestational diabetes mellitus. American Journal of Obstetrics \& Gynecology, 200(6):672-e1.

6- Dudley NJ (2005): A systematic review of the ultrasound estimation of fetal weight. Ultrasound Obstet Gynecol., 25:80.

7- Chudleigh T, Smith A, Cumming $\mathbf{S}$ (2016): Obstetric \& Gynaecological Ultrasound E-Book: How, Why and When. Elsevier Health Sciences.

8- Fu J, Olofsson P (2011): Relations between fetal brain sparing circulation, oxytocin challenge test, mode of delivery and fetal outcome in growth restricted term fetuses. Acta Obstet Gynecol Scand., 90:227- 30 .
9- Iura T, Makinoda S, Fujita S, Matsuzawa S, Waseda T, Ohshima $K$ and Tomizawa $H$ (2005): Analysis of renal artery hemodynamics in normal fetuses using the color Doppler method. Fetal diagnosis and therapy, 20(2): 86-90.

10- Surányi A, Kozinszky Z, Molnár A, Nyári T, Bitó $T$ and Pál A (2013): Placental three-dimensional power Doppler indices in mid-pregnancy and late pregnancy complicated by gestational diabetes mellitus. Prenatal diagnosis, 33(10): 952-958.

11- American Diabetes Association (2016): Standards of medical care in diabetes2016. Diabetes Care, 39(1):S1-S106.

12- Liu F, Liu Y, Lai YP, Gu XN, Liu DM, Yang M (2016): Fetal hemodynamics and fetal growth indices by ultrasound in late pregnancy and birth weight in gestational diabetes mellitus. Chinese medical journal, 129(17):2109.

13- Hadlock FP, Harrist RB, Sharman RS, Deter RL and Park SK (1985): Estimation of fetal weight with the use of head, body, and femur measurements - A prospective study. Am J Obstet Gynecol., 151:3337.

14- De Melo BC, de Amorim MM, Katz L, Coutinho I and Veríssimo $G$ (2010): Uterine artery Doppler in the third trimester of pregnancy and postnatal outcome of patients with severe preeclampsia. Hypertens Pregnancy, 29:135-47.

15- Shabani Zanjani M, Nasirzadeh R, Fereshtehnejad SM, Yoonesi Asl L, Alemzadeh SA and Askari S (2014): Fetal cerebral hemodynamic in gestational diabetic versus normal pregnancies: A Doppler velocimetry of middle cerebral and umbilical arteries. Acta Neurol Belgica, 114:15 23.

16- Li J, Chen YP, Dong YP, Yu CH, Lu YP, Xiao XM and Hocher B (2014): The impact of umbilical blood flow regulation on fetal development differs in diabetic and non-diabetic pregnancy. Kidney and Blood Pressure Research, 39(4): 369-377. 
17- Esakoff TF, Cheng YW, Sparks TN and Caughey AB (2009): The association between birthweight $4000 \mathrm{~g}$ or greater and perinatal outcomes in patients with and without gestational diabetes mellitus. American Journal of Obstetrics \& Gynecology, 200(6):672-e1.

18- Dudley NJ (2005): A systematic review of the ultrasound estimation of fetal weight. Ultrasound Obstetrics Gynecology, 25:80.

19- Ghi T, Contro E, Youssef A, Giorgetta F, Farina A, Pilu G and Pelusi G (2010): Persistence of increased uterine artery resistance in the third trimester and pregnancy outcome. Ultrasound in Obstetrics \& Gynecology, 36(5):577-81.

20- Gluckman PD (1986): The role of pituitary hormones, growth factors and insulin in the regulation of fetal growth. Oxford reviews of reproductive biology, 8:1-60.

21- Leung WC, Lam H, Lee CP and Lao TT (2004): Doppler study of the umbilical and fetal middle cerebral arteries in women with gestational diabetes mellitus. Ultrasound in Obstetrics and
Gynecology: The Official Journal of the International Society of Ultrasound in Obstetrics and Gynecology, 24(5):534537.

22- Quintero-Prado R, Bugatto F, Sánchez-Martín P, Fajardo-Expósito MA, Torrejón $R$ and Bartha JL (2016): The influence of placental perfusion on birthweight in women with gestational diabetes. The Journal of Maternal-Fetal \& Neonatal Medicine, 29(1):32-35.

23- Maruotti GM, Rizzo G, Sirico A, Sarno L, Cirigliano L, Arduini D and Martinelli P (2014): Are there any relationships between umbilical artery Pulsatility Index and macrosomia in fetuses of type I diabetic mothers?. The Journal of Maternal-Fetal \& Neonatal Medicine, 27(17):1776-1781.

24- Verburg BO, Jaddoe VW, Wladimiroff JW, Hofman A, Witteman JC and Steegers EA (2008): Fetal hemodynamic adaptive changes related to intrauterine growth: the Generation R Study. Circulation, 117(5):649-59. 